\title{
A SPECTRAL MAPPING THEOREM FOR SOME REPRESENTATIONS OF COMPACT ABELIAN GROUPS
}

\author{
by SIN-EI TAKAHASI and JYUNJI INOUE \\ (Received 30th January 1990)
}

Dedicated to Professor Chinami Watari on his sixtieth birthday

\begin{abstract}
We show that if $G$ is a compact abelian group and $U$ is a weakly continuous representation of $G$ by means of isometries on a Banach space $X$, then $\sigma(\pi(\mu))=\hat{\mu}(\operatorname{sp}(U))$ holds for each measure $\mu$ in reg $(M(G))$, where $\pi(\mu)$ denotes the generalized convolution operator in $B(X)$ defined by $\pi(\mu) x=\int_{G} U(t) x d \mu(t)(x \in X), \sigma$ the usual spectrum in $B(X), \operatorname{sp}(U)$ the Arveson spectrum of $U, \hat{\mu}$ the Fourier-Stieltjes transform of $\mu$ and $\operatorname{reg}(M(G))$ the largest closed regular subalgebra of the convolution measure algebra $M(G)$ of $G$. $\operatorname{reg}(M(G)$ ) contains all the absolutely continuous measures and discrete measures.
\end{abstract}

1980 Mathematics subject classification (1985 Revision): Primary 46H15; Secondary 47A10.

\section{Introduction and main result}

Let $G$ be a locally compact abelian group and $U$ a weakly continuous representation of $G$ by means of isometries on a Banach space $X$, i.e., a map $U: G \rightarrow B(X)$ satisfying

(i) $U(s+t)=U(s) U(t)$ for all $s, t \in G, U(0)=I$,

(ii) $\|U(s) x\|=\|x\|$ for $s \in G, x \in X$,

(iii) $G \rightarrow X ; s \rightarrow U(s) x$ is weakly continuous for each $x \in X$.

Then this representation induces a continuous algebra homomorphism $\pi$ of the convolution algebra $M(G)$ into $B(X)$ and such a homomorphism is written by $\pi(\mu)=\int_{G} U(t) d \mu(t)$ (cf. [5]). Let $s p(U)$ be the Arveson spectrum of $U$ defined by

$$
s p(U)=\bigcap\left\{Z(f): f \in \operatorname{Ker}\left(\pi \mid L^{1}(G)\right)\right\} .
$$

Here $Z(f)$ denotes the set of zeros of the Fourier transform $\hat{f}$ of $f$. In this setting, Connes [4] proved that for every Dirac measure $\mu$ the spectral mapping theorem (SMT): $\sigma(\pi(\mu))=\overline{\hat{\mu}(s p(U))}$ holds, where $\sigma$ denotes the usual spectrum in $B(X)$ and $\hat{\mu}$ denotes the Fourier-Stieltjes transform of $\mu$. Furthermore D'Antoni, Longo and Zsido [2] proved the SMT for the class of measures whose continuous part belongs to $L^{1}(G)$, the group algebra of $G$. Also, Eschmeier [5] proved the SMT in the case that $U$ is the translation group representation and $X=L^{1}(G)$ or $M(G)$ and the convolution operator induced by $\mu$ has the weak 2-SDP (see [5, Theorem 2]). Here $M(G)$ denotes the Banach 
algebra of all bounded regular complex Borel measures on $G$. Also, since $M(G)$ is a semisimple commutative Banach algebra with identity, it follows from Albrecht's theorem [1] that there exists a largest closed regular subalgebra of $M(G)$, which we denote by $\operatorname{reg}(M(G))$.

With this notation, our main theorem can be stated as follows:

Theorem. If $G$ is a compact abelian group and $\mu \in \operatorname{reg}(M(G))$, we have $\sigma(\pi(\mu))=$ $\overline{\hat{\mu}(s p(U))}$.

Remark. The group algebra $L^{1}(G)$ and the discrete measures $M_{d}(G)$ are regular Banach subalgebras of $M(G)$. Then $L^{1}(G)+M_{d}(G) \subset \operatorname{reg}(M(G))$. In general, $L^{1}(G)+$ $M_{d}(G) \neq \operatorname{reg}(M(G))$. In fact, let us denote by top $(G)$ the class of all locally compact group topologies on $G$ which are equal to or stronger than the original topology on $G$ and denote by $L^{*}(G)$ the closed subalgebra of $M(G)$ generated by $\left\{L^{1}(G, \tau): \tau \in \operatorname{top}(G)\right\}$ as in [6]. Then we have that $L^{1}(G)+M_{d}(G) \subset L^{*}(G) \subset \operatorname{reg}(M(G))$ and $L^{1}(G)+M_{d}(G) \neq L^{*}(G)$ in general (cf. [6], [12]). Thus our result contains the Connes-D'Antoni-Longo-Zsido spectral mapping theorem for the compact case.

\section{Lemmas}

We first present the following result obtained in [7], which plays an essential role in the proof of the main theorem, and we include its proof for completeness.

Lemma 1. Let $X$ be a commutative Banach algebra with identity and $B$ a Banach subalgebra of $X$. If $B$ is regular, then for any $b \in B$ the Gelfand transform of $b$ as an element of $X$ is continuous on the carrier space $\Phi_{X}$ of $X$ in the hull-kernel topology.

Proof. We can assume without loss of generality that $B$ contains the identity of $X$. Then it is sufficient to show that the restriction map $\theta: \Phi_{X} \rightarrow \Phi_{B} ; \phi \rightarrow \phi \mid B$ is continuous in the hull-kernel topology. To do this let $F$ be a closed subset of $\Phi_{B}$ in the hull-kernel topology. Then $\left\{\phi \in \Phi_{X}: \phi \mid \operatorname{ker} F=0\right\}=\theta^{-1}(F)$. Also, since $\operatorname{ker} F \subset \operatorname{ker} \theta^{-1}(F)$, it follows that hul $\left(\operatorname{ker} \theta^{-1}(F)\right) \subset\left\{\phi \in \Phi_{X}: \phi \mid \operatorname{ker} F=0\right\}$. Therefore $\theta^{-1}(F)$ is closed in the hull-kernel topology. In other words, $\theta$ is continuous in this topology.

We will next state the definition of BSE-algebras introduced by the first author and Hatori [10]. Let $A$ be a commutative Banach algebra without order and $M(A)$ the multiplier algebra of $A$. It is well-known that $T \in M(A)$ can be represented as a bounded continuous complex-valued function $\hat{T}$ on $\Phi_{A}$ such that $\widehat{T a}(\phi)=\hat{T}(\phi) \hat{a}(\phi)$ for all $a \in A$ and $\phi \in \Phi_{A}$ (cf. [8]). Set $\hat{M}(A)=\{\hat{T}: T \in M(A)\}$. We also denote by $A^{*}$ the dual space of $A$ and $C_{\mathrm{BSE}}\left(\Phi_{A}\right)$ the set of all continuous complex-valued functions $\sigma$ on $\Phi_{\mathrm{A}}$ which satisfy the following condition: there exists a positive real number $\beta$ such that for every finite sequence of complex numbers $c_{1}, \ldots, c_{n}$ and elements $\phi_{1}, \ldots, \phi_{n}$ of $\Phi_{A}$, the inequality

$$
\left|\sum_{i=1}^{n} c_{i} \sigma\left(\phi_{i}\right)\right| \leqq \beta\left\|\sum_{i=1}^{n} c_{i} \phi_{i}\right\|_{A^{*}}
$$


holds.

Definition. A commutative Banach algebra $A$ without order is said to be BSE if it satisfies the condition $\hat{M}(A)=C_{\mathrm{BSE}}\left(\Phi_{A}\right)$.

By the Bochner-Schoenberg-Eberlein theorem, the group algebra of a locally compact abelian group is BSE (cf. [10]). The following results can be observed in [10].

Lemma 2 ([10, Theorem 4, (ii)]). Let $A$ be a commutative Banach algebra without order, $A^{* *}$ its second dual and $C^{b}\left(\Phi_{A}\right)$ the set of all bounded continuous complex-valued functions on $\Phi_{A}$. Then $C_{\mathrm{BSE}}\left(\Phi_{A}\right)=C^{b}\left(\Phi_{A}\right) \cap\left(A^{* *} \mid \Phi_{A}\right)$.

When a closed ideal $I$ of a commutative Banach algebra $A$ is essential as a Banach $A$-module, that is, $I$ equals the closed linear span of $\{a x: a \in A, x \in I\}$, we call $I$ an essential ideal.

Lemma 3 ([10, Theorem 8, (i)]). Let $A$ be a BSE-algebra with discrete carrier space and $I$ an essential closed ideal of $A$. Then $\hat{M}(A / I)=C_{\mathrm{BSE}}\left(\Phi_{A / I}\right)$, i.e., $A / I$ is BSE, where $A / I$ denotes the quotient algebra of $A$ defined by $I$.

The following lemma also plays an essential role in the proof of the main theorem.

Lemma 4. Let $A$ be a BSE-algebra with discrete carrier space and I a closed ideal of $A$ such that $I^{\sim}=\operatorname{ker}(h u l(I))$ is essential. Then every multiplier on $A / I^{\sim}$ can be lifted as a multiplier on $A$, that is, if $v \in M\left(A / I^{\sim}\right)$ and $\eta$ is the canonical map of $A$ onto $A / I^{\sim}$, then there exists $\mu \in M(A)$ such that $\eta(\mu a)=v \eta(a)$ for all $a \in A$.

Proof. Let $v \in M\left(A / I^{\sim}\right)$ and let $\eta$ be the canonical map of $A$ onto $A / I^{\sim}$. Note that the algebra $A / I^{\sim}$ is semisimple. Then it is sufficient to show that there exists $\mu \in M(A)$ such that $(\eta(\mu a))^{\wedge}=(v \eta(a))^{\wedge}$ for all $a \in A$. Here ${ }^{\wedge}$ denotes the Gelfand transform on $A / I^{\wedge}$. We have

$$
\begin{aligned}
\hat{v} \in \hat{M}\left(A / I^{\sim}\right) & =C_{\mathrm{BSE}}\left(\Phi_{A / I^{\sim}}\right) \quad \text { (by Lemma 3) } \\
& =\left(A / I^{\sim}\right)^{* *} \mid \Phi_{A / I^{\sim}} \quad \text { (by Lemma 2), }
\end{aligned}
$$

so that there exists $H \in\left(A / I^{\sim}\right)^{* *}$ with $\hat{v}=H \mid \Phi_{A / I^{\sim}}$. Then we can find an element $F \in A^{* *}$ such that $\eta^{* *}(F)=H$, since $\eta^{* *}: A^{* *} \rightarrow\left(A / I^{\sim}\right)^{* *}$ is a surjection. We further have by the BSE property of $A$ and Lemma 2 that

$$
\hat{M}(A)=C_{\mathrm{BSE}}\left(\Phi_{A}\right)=A^{* *} \mid \Phi_{A}
$$

Therefore we can find an element $\mu \in M(A)$ such that $\hat{\mu}=F \mid \Phi_{A}$. Let $\phi \in \Phi_{A}$ be such that $\phi \mid I^{\sim}=0$ and $\phi^{\prime}$ the canonical image of $\phi$ in $\Phi_{A / I^{-}}$. Then we have 


$$
\hat{v}\left(\phi^{\prime}\right)=H\left(\phi^{\prime}\right)=\left\langle\phi^{\prime}, \eta^{* *}(F)\right\rangle=\left\langle\eta^{*}\left(\phi^{\prime}\right), F\right\rangle=\langle\phi, F\rangle
$$

and hence for any $a \in A$,

$$
\begin{aligned}
(v \eta(a))^{\wedge}\left(\phi^{\prime}\right) & =\hat{v}\left(\phi^{\prime}\right)(\eta(a))^{\wedge}\left(\phi^{\prime}\right)=\langle\phi, F\rangle \hat{a}(\phi) \\
& =\hat{\mu}(\phi) \hat{a}(\phi)=(\mu a)^{\wedge}(\phi)=\phi^{\prime}(\eta(\mu a)) \\
& =(\eta(\mu a))^{\wedge}\left(\phi^{\prime}\right) .
\end{aligned}
$$

Consequently $(\eta(\mu a))^{\wedge}=(\nu \eta(a))^{\wedge}$ for all $a \in A$.

Lemma 5. If $\mu \in M(G)$, then $\sigma(\pi(\mu)) \subset\left\{\mu^{\vee}(\phi): \phi \in \Phi_{M(G)}\right.$, $\left.\operatorname{Ker} \pi \subset \operatorname{Ker} \phi\right\}$. Here $\mu^{\vee}$ denotes the Gelfand transform of $\mu \in M(G)$.

Proof. Let $\mu \in M(G)$. Then we have that

$$
\begin{aligned}
\sigma_{M(G) / \operatorname{Ker} \pi}(\mu+\operatorname{Ker} \pi) & =(\mu+\operatorname{Ker} \pi)^{\vee}\left(\Phi_{M(G) / \operatorname{Ker} \pi}\right) \\
& =\left\{\mu^{\vee}(\phi): \operatorname{Ker} \pi \subset \operatorname{Ker} \phi\right\} .
\end{aligned}
$$

Also, since $M(G) / \operatorname{Ker} \pi \cong \pi(M(G)) \subset B(X)$, it follows that

$$
\begin{aligned}
\sigma_{M(G) / \operatorname{Ker} \pi}(\mu+\operatorname{Ker} \pi) & =\sigma_{\pi(M(G))}(\pi(\mu)) \\
& \supset \sigma_{B(X)}(\pi(\mu)) .
\end{aligned}
$$

Therefore the desired inclusion follows.

The following result was proved by D'Antoni, Longo and Zsido [2].

Lemma $6([2$, Lemma 1$]) . \quad \sigma(\pi(\mu)) \supset \overline{\hat{\mu}(s p(U))}$ for all $\mu \in M(G)$.

In the next section we will show our main theorem using these lemmas.

\section{Proof of theorem}

Since $\overline{\hat{\mu}(s p(U))} \subset \sigma(\pi(\mu))$ by Lemma 6 , we have only to show the reverse inclusion. To do this, let $\alpha \in \sigma(\pi(\mu))$. Then by Lemma 5 , there exists $\phi_{0} \in \Phi_{M(G)}: \alpha=\mu^{\vee}\left(\phi_{0}\right)$ and $\operatorname{Ker} \pi \subset \operatorname{Ker} \phi_{0}$.

Let us consider the natural homomorphism $T_{\pi}$ of $M(G) / \operatorname{Ker} \pi$ into $M\left(L^{1}(G) / I_{n}\right)$ defined by

$$
T_{\pi}(v+\operatorname{Ker} \pi)\left(f+I_{\pi}\right)=v * f+I_{\pi} \quad\left(v \in M(G), f \in L^{1}(G)\right)
$$


where $I_{\pi}=\operatorname{Ker}\left(\pi \mid L^{1}(G)\right)$.

Since $G$ is compact, it follows from [9, Corollary 8.3.2] that $I_{\pi}^{\sim}=I_{\pi}$. Note also that $L^{1}(G)$ is a BSE-algebra with discrete carrier space and it has an approximate identity; hence $I_{\pi}$ is an essential ideal of $L^{1}(G)$. Then Lemma 4 implies that $T_{\pi}$ is surjective, since $M(G) \cong M\left(L^{1}(G)\right)$. Furthermore, $T_{\pi}$ is injective. In fact, let $v \in M(G)$ be such that $\pi(v * f)=0$ for all $f \in L^{1}(G)$. Given $\varepsilon>0, x \in X$ and $\xi \in X^{*}$, the dual space of $X$, choose a neighbourhood $V$ of zero such that

$$
\left|\left\langle U(t) x,(\pi(v))^{*} \xi\right\rangle-\left\langle x,(\pi(v))^{*} \xi\right\rangle\right|<\varepsilon \quad(t \in V) .
$$

Furthermore, choose a non-negative real-valued function $u_{V} \in L^{1}(G)$ vanishing off $V$ and satisfying $\int_{G} u_{V}(t) d t=1$. Then we have

$$
\begin{aligned}
|\langle\pi(v) x, \xi\rangle| & \leqq\left|\left\langle\pi\left(u_{V}\right) x,(\pi(v))^{*} \xi\right\rangle-\left\langle x,(\pi(v))^{*} \xi\right\rangle\right|+\left|\left\langle\pi\left(u_{V}\right) x,(\pi(v))^{*} \xi\right\rangle\right| \\
& \leqq \int_{V}\left|\left\langle U(t) x,(\pi(v))^{*} \xi\right\rangle-\left\langle x,(\pi(v))^{*} \xi\right\rangle\right| u_{V}(t) d t \\
& <\varepsilon .
\end{aligned}
$$

Since $\varepsilon$ is arbitrary, it follows that $\langle\pi(v) x, \xi\rangle=0$ for all $x \in X$ and $\xi \in X^{*}$; hence $\pi(v)=0$. In other words, $T_{\pi}$ is injective.

Here we take the following convention: for each $\phi \in \Phi_{M(G)}$ such that $\operatorname{Ker} \pi \subset \operatorname{Ker} \phi, \phi^{\prime}$ denotes the element of $\Phi_{M(G) / K e r \pi}$ defined by $\phi^{\prime}(v+\operatorname{Ker} \pi)=\phi(v)(v \in M(G))$.

Since $T_{\pi}$ is an isomorphism of $M(G) / \operatorname{Ker} \pi$ onto $M\left(L^{1}(G) / I_{\pi}\right)$, there exists an element $\psi_{0}$ of $\Phi_{M\left(L^{\prime}(G) / I_{k}\right)}$ such that $\phi_{0}^{\prime}=\left(T_{\pi}\right)^{*} \psi_{0}$. So we can find a net $\left\{\psi_{\lambda}\right\}$ in $\Phi_{M\left(L^{\prime}(G) / I_{*}\right)}$ such that $\psi_{\lambda} \mid L^{1}(G) / I_{\pi} \neq 0$ for all $\lambda$ and $h k-\lim \psi_{\lambda}=\psi_{0}$, where " $h k$-lim" denotes the hull-kernel limit. Furthermore, we can find a net $\left\{\phi_{\lambda}\right\}$ in $\Phi_{M(G)}$ such that $\operatorname{Ker} \pi \subset \operatorname{Ker} \phi_{\lambda}$ and $\left(T_{\pi}\right)^{*} \psi_{\lambda}=\phi_{\lambda}^{\prime}$ for all $\lambda$. Set $\xi_{\lambda}=\phi_{\lambda} \mid L^{1}(G)$ for each $\lambda$. Then each $\xi_{\lambda} \neq 0$. In fact, choose a function $f_{0} \in L^{1}(G)$ such that $\psi_{\lambda}\left(f_{0}+I_{\pi}\right) \neq 0$. Then for each $\lambda$, we have

$$
\phi_{\lambda}\left(f_{0}\right)=\phi_{\lambda}^{\prime}\left(f_{0}+I_{\pi}\right)=\left\langle T_{\pi}\left(f_{0}+I_{n}\right), \psi_{\lambda}\right\rangle=\psi_{\lambda}\left(f_{0}+I_{n}\right) \neq 0
$$

so that $\xi_{\lambda} \neq 0$. Thus each $\xi_{\lambda}$ belongs to $\Phi_{L^{1}(G)}(\cong \hat{G}$, the dual group of $G)$ and hence must belong to $s p(U)$, since $I_{\pi} \subset \operatorname{Ker} \xi_{\lambda}$ and $s p(U)$ can be regarded as the hull of $I_{\pi}$ in $\Phi_{L^{1}(G)}$.

Of course $\left(T_{n}\right)^{*} \mid \Phi_{M\left(L^{1}(G) / I_{x}\right)}$ is continuous on $\Phi_{M\left(L^{\prime}(G) / I_{t}\right)}$ in the hull-kernel topology and hence

$$
h k-\lim \phi_{\lambda}^{\prime}=h k-\lim \left(T_{\pi}\right)^{*} \psi_{\lambda}=\left(T_{\pi}\right)^{*} \psi_{0}=\phi_{0}^{\prime}
$$

Therefore we have from [11, Theorem 2.6.6] that $h k-\lim \phi_{\lambda}=\phi_{0}$. Since $\mu \in \operatorname{reg}(M(G))$, it follows from Lemma 1 that $\mu^{v}$ is continuous on $\Phi_{M(G)}$ in the hull-kernel topology, so that

$$
\lim \hat{\mu}\left(\xi_{\lambda}\right)=\lim \mu^{\vee}\left(\phi_{\lambda}\right)=\mu^{\vee}\left(\phi_{0}\right)=\alpha
$$


Consequently we have that $\alpha \in \overline{\hat{\mu}(s p(U))}$ and the reverse inclusion is shown.

\section{REFERENCES}

1. E. Albrecht, Decomposable systems of operators in harmonic analysis, in Toeplitz Centennial (I. Goldberg (ed.), Birkhauser, Basel, 1982), 19-35.

2. C. D'Antoni, R. Longo and L. Zsido, A spectral mapping theorem for locally compact groups of operators, Pacific J. Math. 103 (1981), 17-24.

3. W. Arveson, On groups of automorphisms of operator algebras, J. Funct. Anal. 15 (1974), 217-243. 133.

4. A. Connes, Une classification des facteurs de type III, Ann. Sci. Ecole Norm. Sup. 6 (1973),

5. J. EschmeIeR, Operator decomposability and weakly continuous representations of locally compact abelian groups, J. Operator Theory 7 (1982), 201-208.

6. J. INoue, Some closed subalgebras of measure algebras and a generalization of P. J. Cohen's theorem, J. Math. Soc. Japan 23 (1971), 278-294.

7. J. InOuE and S.-E. TAKAHASI, A remark on the largest regular subalgebra of a Banach algebra, Proc. Amer. Math. Soc., to appear.

8. R. LARSEN, An introduction to the theory of multipliers (Springer-Verlag, New YorkHeidelberg, 1971).

9. R. LaRsen, Banach Algebras (Marcel Dekker, Inc., New York, 1973).

10. S.-E. TAKahasi and O. Hatori, Commutative Banach algebras which satisfy BochnerSchoenberg-Eberlein type theorem, Proc. Amer. Math. Soc. 110 (1990), 149-158.

11. C. E. Rickart, General Theory of Banach Algebras (D. Van Nostrand, Princeton, N.J., 1960).

12. N. W. Rickert, Locally compact topologies for groups, Trans. Amer. Math. Soc. 119 (1965), 150-166.

Department of Basic Technology

Applied Mathematics and Physics

YAMAGATA UNIVERSITY

Yonezawa 992, Japan
Department of Mathematics

HokKaIDo UNIVERSITY

SAPPORO 060, JaPAN 